\title{
Guest Editorial: Shape Analysis Beyond the Eikonal Equation
}

\author{
Sibel Tari ${ }^{1}$ - Luminita Vese ${ }^{2}$ - Anand Rangarajan ${ }^{3}$
}

Published online: 10 March 2016

(C) Springer Science+Business Media New York 2016

"Shape Analysis" is an interesting research area which contains many thought-provoking problems and mathematical challenges, especially at the representation level. Shape of an object is considered as the enclosing surface (the boundary) or the enclosed interior. One of the major challenges is to define representations of "shapes" that can be manipulated by image processing methods. This is an important need in practice because more often than not shapes need to be extracted or recovered from raw images; moreover, the process of recovering shapes from raw images highly benefits from prior knowledge of candidate shapes.

One of the key means of shape representation is the Euclidean Distance Transform (EDT), which is governed by the Eikonal Equation. The values of the EDT at each interior point give the distance to the nearest boundary point. This distance notion is used in almost any problem that requires integrating shape and image information; e.g., a rich variety of shape-guided image processing or shape extraction tasks. Especially in the 1990s, the idea of embedding shapes as zeros of the signed EDT is highly popularized by the level-set

Sibel Tari

stari@metu.edu.tr

Luminita Vese

lvese@math.ucla.edu

Anand Rangarajan

anand@cise.ufl.edu

1 Department of Computer Engineering, Middle East Technical University, 06800 Ankara, Turkey

2 Department of Mathematics, University of California Los Angeles, Los Angeles, CA 90095, USA

3 Department of Computer and Information Science and Engineering, University of Florida, Gainesville, FL 32611, USA framework and its fast implementation, leading to a booming research area in implicit shape representations. However, as challenging problems are developed, formulations that move beyond the classical Eikonal Equation emerged. This special issue consists of six papers each exploring an interesting direction moving beyond the Eikonal formulation in the field of Shape Analysis.

The first two papers, "Analysis and Approximation of Some Shape-from-Shading Models for non-Lambertian Surfaces" (doi:10.1007/s10851-016-0636-x) and "A Unified Variational Volume Registration Method based on Automatically Learned Brain Structures" (doi:10.1007/ s10851-015-0604-x), deal with two key applications that jointly involve shapes and images. In the first paper, Silvia Tozza and Maurizio Falcone present a unified mathematical formulation of some non-Lambertian models, which lead to more complex stationary nonlinear partial differential equations of Hamilton-Jacobi type which can be regarded as a generalization of the classical Eikonal Equation corresponding to the Lambertian case. In the second paper, Carl Lederman et al. present an active volume technique employing level sets of the distance function. Unlike the common practice, the level sets in their formulation are only utilized on their respective regions and not on the whole domain, making the computations feasible as the number of regions increases.

The next four papers propose alternative distance notions or computations. They are briefly discussed as follows.

In "Affine Invariant Distance Using Multiscale Analysis" (doi:10.1007/s10851-015-0585-9), Luis Alvarez et al. present a distance transform for planar shapes that is invariant under any planar projection of the shape. Their model is based on partial differential equations which include power function of the image level set curvature. 
In "Geodesic Distance and Curves through Heat Equations on Images and Surfaces" (doi:10.1007/s10851-015-0621-9), Fang Yang and Laurent Cohen investigate the use of modified diffusion flows for tracking stable geodesics of image structures.

In "Nonlocal discrete $\infty$-Poisson and Hamilton Jacobi equations" (doi:10.1007/s10851-015-0592-x), Matthieu Toutain et al. present a new method for computing a distance function on discrete data represented as a weighted graph via adapting the $\infty$-Poisson.
Finally, in "NonLocal via Local-NonLinear via Linear: A New Part-coding Distance Field via Screened Poisson Equation" (doi:10.1007/s10851-015-0614-8), Murat Genctav et al. present an unconventional distance field with nonlocal and nonlinear features by simply solving a local and linear equation twice. 